\title{
CRESCIMENTO E TEORES NUTRICIONAIS DE PARAPIPTADENIA RIGIDA CONSORCIADO COM PASTAGEM EM DIFERENTES ESPAÇAMENTOS E ADUBAÇÃO
}

\section{GROWTH AND NUTRITIONAL CONTENTS OF PARAPIPTADENIA RIGIDA COMBINED WITH PASTURE IN DIFFERENT SPACINGS AND FERTILIZATION}

\author{
Matheus Teixeira Martins ${ }^{1}$, Monique Pimentel Lagemann ${ }^{2}$, Hamilton Luiz Munari Voge ${ }^{3}$, \\ Cristhian Augusto Bugs ${ }^{4}$, Emanuel Arnoni Costa ${ }^{5}$, Mauro Valdir Schumacher ${ }^{6}$ \\ 1, 3,4 Universidade Federal do Pampa, São Gabriel, RS, Brasil - mteixeiramartins@gmail.com, \\ hamiltonvogel@yahoo.com.br \& cristhianbugs@gmail.com \\ 2, 6 Universidade Federal de Santa Maria, Santa Maria, RS, Brasil - moniquelagemann@gmail.com \& \\ mvschumacher@gmail.com
}

${ }^{5}$ Universidade Federal de Uberlândia, Monte Carmelo, MG, Brasil - emanuelarnonicost@hotmail.com

RESUMO

A definição de práticas silviculturais que proporcionem crescimento e a nutrição das espécies em sistema de consórcio, auxilia na adoção desses sistemas e promove a recuperação da qualidade ambiental e produtiva. Diante disso, objetivou-se com este estudo avaliar o crescimento inicial e os teores nutricionais de Parapiptadenia rigida (Benth.) Brenan consorciada com pastagem nativa em São Gabriel, RS. O experimento foi conduzido em delineamento em blocos ao acaso com parcelas subdivididas, avaliando-se diferentes arranjos espaciais (4 níveis) e adubação (2 níveis). As variáveis dendrométricas foram mensuradas aos 24 meses de idade. Para a análise do estado nutricional de macro e micronutrientes foram coletadas amostras compostas das folhas de cinco árvores de diâmetro médio por tratamento, no terço médio da copa, nos quatro quadrantes. Para pastagem, foi coletada toda biomassa com dez repetições por tratamento. A adubação de pastagem promoveu o maior crescimento inicial de $P$. rigida, no entanto nos arranjos espaciais não foram verificadas respostas de crescimento. Os diferentes tratos culturais do sistema não influenciaram significativamente o estado nutricional do angico-vermelho e da pastagem nativa.

PALAVRAS-CHAVE: Espécie nativa, Nutrição florestal, Silvicultura.

\section{ABSTRACT}

The definition of silvicultural practices that provide growth and nutrition of species in an intercrop system assists in the adoption of these systems and promotes the recovery of environmental and productive quality. The objective of this study is to evaluate if different system treatments, fertilization and spatial arrangement influence the initial development and nutritional aspects in a Parapiptadenia rigida (Benth.) Brenan plantation intercropped with native pasture in São Gabriel, RS. The experiment was conducted in a randomized block design with split plots, with different spatial arrangements (4 levels) and fertilization (2 levels). Dendrometric variables were measured from all tree individuals at 24 months of age. For the analysis of the nutritional status of macro and micronutrients, samples were collected from the leaves of five average diameter trees per treatment, in the middle third of the crown, in the four quadrants. For pasture, all biomass were collected, with ten replications per treatment. Pasture fertilization promoted the highest initial growth of $P$. rigida, while in the different spatial arrangements no growth responses were observed. The different cultural treatments of the system did not affect the nutritional status of angico-vermelho and native pasture.

KEYWORDS: Native species, Forest nutrition, Silviculture. 


\section{INTRODUÇÃO}

A pecuária como principal atividade produtiva no bioma Pampa tem ocasionado problemas ambientais. Entre eles estão a degradação de pastagens, erosão dos solos, assoreamento rios (RIBASKI et al., 2005) e perda de biodiversidade florística (BOLDRINI, 2009). Por conseguinte, atualmente as áreas de pastagem nativa apresentam elevada invasão por de espécies exóticas (GUADAGNIN et al., 2009), declínio da produtividade e substituição por monocultivos florestais (RIBASKI et al., 2005).

Os consórcios de espécies arbóreas com pastagens são uma alternativa para a superação destes problemas, uma vez que a integração tem se provado ambientalmente sustentável (JOSE \& DOLLINGER, 2019). Isso se deve ao fato dos consórcios promoverem a melhoria da qualidade do solo (MARTíNEZ et al., 2014), redução da erosão, redução e mitigação de gases do efeito estufa (PORFÍRIODA-SILVA et al., 2010), e aumento no estoque de carbono no solo e na biomassa (LÓPEZ-SANTIAGO et al., 2018). Tais benefícios ambientais repercutem em ganhos econômicos pela produtividade animal (CASTRO et al., 2008) e vegetal (PACIULLO et al., 2011).

A Parapiptadenia rigida (Benth.) Brenan, conhecida como angico-vermelho, é uma Fabaceae arbórea nativa da Floresta Estacional Semidecidual, com ampla distribuição nos estados sulinos (LORENZI, 2008). A espécie é pioneira, fixadora de nitrogênio, com boa deposição de folhedo, o que a torna indicada para recuperação de áreas degradadas e enriquecimento nutricional de ecossistemas (VOGEL et al., 2012). Além disso, apresenta potencial uso madeireiro, por ser caracterizada como muito pesada, elástica e bastante durável (BORGO et al., 2011), e não-madeireiros, como o tanino (SILVA et al., 2017).

Na silvicultura é necessária a adequada definição do espaçamento de plantio para uma espécie florestal. O espaçamento de plantio interfere na taxa de crescimento das plantas, na utilização de recursos, no recobrimento do solo, no manejo, na quantidade e na qualidade de biomassa produzida (NASCIMENTO et al., 2012; LELES et al., 2013). Já a prática silvicultural da adubação, além de suprir nutrientes limitantes, aumenta a produção de biomassa e estoques de nutrientes (RANGELVASCONCELOS et al., 2016).

Apesar da sua importância, a silvicultura de espécies nativas ainda carece de informações (NASCIMENTO et al., 2012). Sobretudo, informações quanto as suas relações ecológicas, seja em plantios puros ou consorciados (BRUN et al., 2013), e referentes aos aspectos nutricionais no sistema solo-planta (VIERA \& SCHUMACHER, 2011).

Levando em consideração o estabelecimento de consórcios de espécies arbóreas nativas e pastagem, torna-se notável a necessidade de se avaliar os tratos culturais que assegurem qualidade dos componentes integrantes. Dessa forma, objetivou-se com este estudo avaliar o crescimento inicial e aspectos nutricionais de $P$. rigida consorciada com pastagem nativa no munícipio de São Gabriel, RS.

\section{MATERIAL E MÉTODOS}

\section{Descrição da área experimental e implantação do experimento}

A área experimental localizada na FEPAGRO Forrageiras, no município de São Gabriel-RS, apresenta uma área de 3,2 ha, caracterizada pela vegetação campestre natural em grau médio de alteração devido ao histórico manejo de cultivos e pastoreio extensivo com bovinos. As principais espécies nativas de valor forrageiro encontradas na área foram: Paspalum plicatulum Michx., Axono pusaffinis Chase, Paspalum notatum Fluegge e Desmodium incanum DC. As principais espécies indesejáveis encontradas na área foram: Eragrostis plana Ness e Eupatorium buniifolium Hook. Ex Arn.

O clima da região é do tipo Cfa, subtropical úmido com verão quente, segundo classificação de Köppen, com uma precipitação total média anual de $1424 \mathrm{~mm}$ e temperatura média anual de 19,5 ㅇ (ALVARES et al., 2013). O solo do local é classificado como um Argissolo Vermelho Distrófico Latossólico, com saturação de bases $<50 \%$ e $B$ latossólico abaixo do $B$ textural de argila dentro de $150 \mathrm{~cm}$ da superfície do solo (EMBRAPA, 2018).

O solo apresenta baixa fertilidade, de acordo com a análise de solo (Tabela 1) e interpretação dos resultados (CQFS, 2016). As camadas de $0-20 \mathrm{~cm}$ e $20-40 \mathrm{~cm}$, respectivamente, apresentaram: teores médio e baixo de matéria orgânica no solo; valores baixos de $\mathrm{pH}$; teores baixos de $\mathrm{K}$ trocável e $\mathrm{P}$ disponível; teores médios de $\mathrm{Ca}$ trocável e $\mathrm{Mg}$ trocável. A saturação por alumínio foi menor que $20 \%$ e saturação por bases até $34 \%$, em ambas as camadas avaliadas.

As mudas de $P$. rigida utilizadas no plantio foram produzidas no viveiro florestal da FEPAGRO Florestas, em Santa Maria, RS. Na implantação, a calagem e adubação de plantio foram estabelecidas com base na análise de solo (Tabela 1) e conforme recomendação para a espécie Mimosa scrabella Benth (bracatinga), de acordo com o 
Manual de Calagem e Adubação para os Estados do RS e SC (CQFS, 2016). A utilização das recomendações para bracatinga se deve por ser da mesma família (Fabaceae) e subfamília (Mimosoideae) botânica que angico-vermelho.

Tabela 1. Caracterização do solo da área experimental no município de São Gabriel, Rio Grande do Sul.

\begin{tabular}{|c|c|c|c|c|c|c|}
\hline $\begin{array}{c}\text { Camada } \\
(\mathrm{cm})\end{array}$ & Argila & M.O. & $\begin{array}{c}\mathrm{pH} \\
\mathrm{H}_{2} \mathrm{O} \\
\end{array}$ & $\begin{array}{c}\text { Índice } \\
\text { SMP }\end{array}$ & $\begin{array}{l}\mathbf{P} \\
(\mathrm{m}\end{array}$ & $\begin{array}{r}\mathbf{K} \\
\left.\mathrm{m}^{-3}\right) \\
\end{array}$ \\
\hline $0-20$ & 23 & 2,8 & 5,2 & 5,5 & 5,6 & 39 \\
\hline $20-40$ & 34 & 2 & 5,1 & 5,5 & 3,6 & 25 \\
\hline $\begin{array}{c}\text { Camada } \\
(\mathrm{cm})\end{array}$ & \multicolumn{3}{|c|}{$\left(\mathrm{cmol}_{\mathrm{c}} \mathrm{dm}^{-3}\right)$} & CTC $_{\text {eft. }}$ & $\mathbf{v}$ & \%) \\
\hline $0-20$ & 0,4 & 3,1 & 1 & 12,5 & 34 & 7,8 \\
\hline $20-40$ & 0,6 & 3 & 0,8 & 11,6 & 33 & 13,4 \\
\hline
\end{tabular}

O plantio das mudas de angico-vermelho ocorreu em outubro de 2012. A adição da adubação foi através da retirada do solo correspondente ao volume da cova $\left(0,027 \mathrm{~m}^{3}\right)$, com a homogeneização do adubo e do solo, o qual posteriormente foi recolocado na cova no momento do plantio das mudas. As quantidades de adubos aplicados, por cova, foram $25 \mathrm{~g}$ de $\mathrm{N}, 84 \mathrm{~g}$ de $\mathrm{P}_{2} \mathrm{O}_{5}$ e $87 \mathrm{~g}$ de $\mathrm{K}_{2} \mathrm{O}$.

O delineamento experimental utilizado foi de blocos ao acaso, com três repetições e com quatro parcelas de $30 \mathrm{~m}$ de comprimento $\times 36 \mathrm{~m}$ de largura por bloco (Figura 1), onde cada parcela apresentava diferentes arranjos espaciais (espaçamento) de plantio: Pastagem nativa (T1) - Somente pastagem nativa, sem plantio de angicovermelho (testemunha); 2x4 (T2) - Plantio de angicovermelho com arranjo espacial de $2 \mathrm{~m} \times 4 \mathrm{~m}$, linhas simples (144 árvores por parcela); 10(2×2)(T3) Implantação de angico-vermelho com arranjo espacial de linhas duplas $2 \mathrm{~m} \times 2 \mathrm{~m}$, a cada $10 \mathrm{~m}$ (96 árvores por parcela); 6(2×2)(T4) - Implantação de angico-vermelho com arranjo espacial de linhas duplas $2 \mathrm{~m} \times 2 \mathrm{~m}$, a cada 6 $\mathrm{m}$ (128 árvores por parcela). As parcelas foram divididas em duas subparcelas de $15 \mathrm{~m}$ de comprimento $\times 36 \mathrm{~m}$ de largura, que receberam os tratamentos com e sem adubação de pastagem.

A adubação de cobertura para pastagem nativa foi realizada conforme a Recomendação de Adubação e Calagem para os Estados do RS e SC (CQFS, 2016) para "Consorciações de gramíneas e leguminosas de Estação Fria". Foram aplicados anualmente nas subparcelas com adubação $50 \mathrm{~kg} \mathrm{ha}^{-1}$ de N, $40 \mathrm{~kg} \mathrm{ha}^{-1}$ de $\mathrm{P}_{2} \mathrm{O}_{5}$, e $70 \mathrm{~kg} \mathrm{ha}^{-1}$ de $\mathrm{K}_{2} \mathrm{O}$. Essas adubações de cobertura foram realizadas no início da primavera com aplicação à lanço.
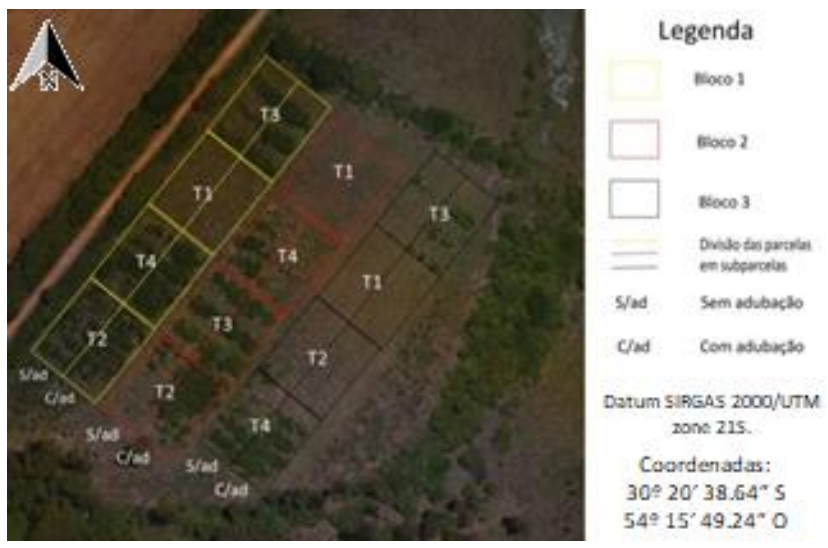

Figura 1. Espacialização dos blocos e tratamentos a parir de imagem obtida com drone em 2019 na área experimental, no município de São Gabriel-RS.

\section{Avaliação de crescimento e estado nutricional}

Aos 24 meses idade, foram mensurados a altura e diâmetro a altura do colo (DAC) de todos os indivíduos (1104 árvores) com utilização de régua graduada $\mathrm{e}$ paquímetro digital, respectivamente.

No mesmo período, o estado nutricional das árvores foi determinado por meio da coleta de amostras compostas, contendo as folhas de cinco árvores de diâmetro médio, por tratamento, no terço médio da copa e nos quatro quadrantes, aproximadamente $100 \mathrm{~g}$ de folha por árvore foram coletadas. Para a pastagem nativa, toda a biomassa contida no interior do gabarito metálico $\left(0,25 \mathrm{~m}^{2}\right)$, distribuído de forma aleatória na parcela, foi removida com o auxílio de uma tesoura elétrica, sendo 10 repetições por tratamento.

Em laboratório, as amostras de tecido vegetal tanto do do componente arbóreo quanto da pastagem, foram secas em estufa de circulação e renovação de ar a $70^{\circ} \mathrm{C}$ até atingir o peso constante, por $72 \mathrm{~h}$. Logo após a secagem, as amostras foram moídas em moinho de lâminas do tipo Wiley, com peneira de 30 mesh. Determinaram-se os teores de nitrogênio $(N)$ pelo método Kjeldahl; fósforo (P) e boro (B) por espectrometria visível; potássio (K) por fotometria de chama; enxofre (S) por turbidimetria, cálcio $(\mathrm{Ca})$, magnésio $(\mathrm{Mg})$, cobre $(\mathrm{Cu})$, ferro (Fe), manganês (Mn) e zinco (Zn) por espectrofotometria de absorção atômica, seguindo a metodologia descrita por Tedesco et al. (1995).

\section{Análise estatística}

A análise estatística dos dados obtidos foi realizada por meio do software $R$ versão 3.2.4 (R CORE TEAM, 2016). Os dados foram submetidos aos testes de Bartlett 
para verificação da homogeneidade da variância e de Shapiro-Wilk para normalidade.

Seguindo, realizou-se a análise de variância para as variáveis altura, DAC e teores foliares de nutrientes do angico-vermelho e da pastagem nativa nos diferentes tratamentos. Os testes adotados para a comparação múltipla de médias foi teste Tukey e teste $t$ de Student, ambos a $5 \%$ de probabilidade de erro.

\section{RESULTADOS E DISCUSSÃO}

\section{Crescimento inicial de $P$. rigida}

Nas subparcelas onde se realizou a adubação de cobertura para pastagem nativa proporcionou crescimento superior em altura e DAC de $P$. rigida (Tabela 2). Em média, as árvores adubadas obtiveram $1,55 \mathrm{~m}$ de altura, um aumento de $32,5 \%$ em comparação a altura média das árvores não adubadas. Já para o diâmetro médio das árvores adubadas o aumento foi 20,1\% em relação às árvores sem adubação.

Tabela 2. Altura e diâmetro a altura do colo (DAC) médio das árvores de $P$. rigida nos diferentes tratamentos, aos 24 meses de idade.

\begin{tabular}{cccc}
\hline $\begin{array}{c}\text { Arranjo } \\
\text { Espacial }\end{array}$ & Adubação & $\begin{array}{c}\text { Altura } \\
(\mathbf{m})\end{array}$ & $\begin{array}{c}\text { DAC } \\
(\mathbf{c m})\end{array}$ \\
\hline $\mathrm{T} 2$ & Sem & $1,20^{\mathrm{BCa}}$ & $2,89 \mathrm{BCa}$ \\
$\mathrm{T} 2$ & $\mathrm{Com}$ & $1,68^{\mathrm{Ab}}$ & $3,58 \mathrm{Ab}$ \\
$\mathrm{T} 3$ & $\mathrm{Sem}$ & $1,06 \mathrm{Ca}$ & $2,56 \mathrm{Ca}$ \\
$\mathrm{T} 3$ & $\mathrm{Com}$ & $1,44 \mathrm{ABb}$ & $3,09 \mathrm{ABCb}$ \\
$\mathrm{T} 4$ & Sem & $1,26 \mathrm{BCa}$ & $2,93 \mathrm{BCa}$ \\
$\mathrm{T} 4$ & Com & $1,54 \mathrm{Ab}$ & $3,37 \mathrm{ABb}$ \\
\hline
\end{tabular}

(1) Letras maiúsculas referem-se ao teste de Tukey (arranjo espacial) e letras minúsculas referem-se ao teste $t$ Student (adubação) para separação dos contrastes de médias.

Em viveiro, $P$. rigida alguns trabalhos já demonstram a resposta à adubação NPK, verificada pelo maior crescimento em altura e DAC (SCHEER et al., 2010; GASPARIN et al., 2017). No entanto, à campo a fertilização assegura maiores taxas de sobrevivência da espécie, evitando elevadas taxas de mortalidade quando essa prática não é realizada (GASPARIN et al., 2017). Assim o nosso estudo apresenta a resposta no crescimento inicial a da espécie quando submetida a prática da adubação a campo, resultados na literatura obtidos até então só em viveiro (SCHEER et al., 2010; GASPARIN et al., 2017).

Em relação aos arranjos espaciais, $P$. rigida obteve crescimento superior nas variáveis mensuradas no arranjo espacial $2 \times 4$ (T2), mas sem diferir dos demais arranjos espaciais com adubação. Outro trabalho obteve maior crescimento da espécie em espaçamentos de plantio mais amplos, alcançando uma altura de $2,8 \mathrm{~m}$ e DAC de 5,91 $\mathrm{cm}$ aos 22 meses de idade no espaçamento de $3 \mathrm{~m} \times 2 \mathrm{~m}$ (NASCIMENTO et al., 2012).

Ao avaliar o efeito da utilização de plantas de cobertura fixadoras de $\mathrm{N}$, como Vicia sativa (ervilhaca) na melhoria nutricional em plantios de recuperação de áreas degradadas com $P$. rigida em espaçamento $1,5 \mathrm{~m} \times 1,5 \mathrm{~m}$, não houve resposta no crescimento da espécie florestal (MARCUZZO et al., 2015). Em nível de comparação os autores obtiveram $97,8 \mathrm{~cm}$ de altura e $1,19 \mathrm{~cm}$ de DAC aos 24 meses de idade.

Ao contrário dos resultados da literatura, nosso trabalho demonstra o efeito das práticas silviculturais no crescimento da espécie nativa. Uma vez que $P$. rigida no arranjo espacial $2 \times 4$ (T2) com área útil de $8 \mathrm{~m}^{2}$ planta $^{-1}$, com maior espaçamento interespecífico, associado a adubação, foram essenciais para o maior crescimento inicial da espécie.

Isso se explica pela relação linear existente entre aumento de área útil por planta e crescimento em diâmetro nos estágios iniciais de crescimento de espécies florestais (OLIVEIRA NETO et al., 2010). Ainda, torna-se essencial utilização de espaçamentos mais amplos para propiciar maior diâmetro, ao considerar o componente arbóreo do sistema para fins madeireiro e de serraria (LIMA et al., 2013).

Levantamos a hipótese de que a prática de adubação melhorou a qualidade nutricional do sítio pelo aporte de nutrientes, possibilitando o maior crescimento arbóreo. Reforçamos essa hipótese pelo fato de que locais onde os teores de matéria orgânica são baixos, o crescimento vegetal só consegue ser satisfatório através da utilização de insumos como fertilizantes (FRANCO et al., 2003). Considerando os teores baixos de matéria orgânica do solo na área do experimento nos primeiro $20 \mathrm{~cm}$ de solo e a saturação por bases $<50 \%$ (Tabela 1 ), torna-se evidente a importância da fertilização fornecimento de nutrientes para o sistema.

Contudo, ambas as prática devem ser avaliadas ao longo do tempo na averiguação da sua eficiência no crescimento. Ainda, estudos de ciclagem de nutrientes tornam-se imprescindíveis para estabelecer a idade limite para realização da prática da adubação.

\section{Análise nutricional de $P$. rigida e pastagem nativa}

De forma geral os tratamentos avaliados, adubação e 
arranjo espacial, não influenciaram em diferenças nos teores nutricionais de $P$. rigida e da pastagem nativa.

Os teores de macronutrientes nas folhas de angicovermelho não apresentaram diferença significativa entre os tratamentos avaliados (Tabela 3). Comportamento semelhante foi obtido para os teores de macronutrientes da biomassa da pastagem nativa. Os macronutrientes tanto de $P$. rigida quanto da pastagem nativa seguiram a tendência de concentração decrescente $\mathrm{N}>\mathrm{K}>\mathrm{Ca}>\mathrm{Mg}>\mathrm{P}>\mathrm{S}$.

Tabela 3. Teores médios de macronutrientes nas folhas de $P$. rigida e na biomassa da pastagem nativa nos diferentes tratamentos, aos 24 meses de idade.

\begin{tabular}{|c|c|c|c|c|c|c|c|}
\hline \multirow{2}{*}{$\mathrm{AE}$} & \multirow{2}{*}{ A } & $\mathbf{N}$ & $\mathbf{P}$ & K & $\mathrm{Ca}$ & $\mathrm{Mg}$ & $S$ \\
\hline & & \multicolumn{6}{|c|}{$\left(\mathrm{g} \mathrm{kg}^{-1}\right)$} \\
\hline \multicolumn{8}{|c|}{$P$. rigida } \\
\hline $\mathrm{T} 2$ & C & $24,4^{\mathrm{Aa}^{1}}$ & $1,4^{\mathrm{Aa}}$ & $8,3^{\mathrm{Aa}}$ & $5,7^{\mathrm{Aa}}$ & $2,1^{\mathrm{Aa}}$ & $1,2^{A} \bar{c}$ \\
\hline $\mathrm{T} 2$ & $S$ & $23,4^{\mathrm{Aa}}$ & $1,3^{\mathrm{Aa}}$ & $7,3^{\mathrm{Aa}}$ & $5,1^{\mathrm{Aa}}$ & $1,9 \mathrm{Aa}$ & $1,1^{A c}$ \\
\hline T3 & C & $23,0^{\mathrm{Aa}}$ & $1,5^{\mathrm{Aa}}$ & $8,8^{\mathrm{Aa}}$ & $6,8^{\mathrm{Aa}}$ & $2,5^{\mathrm{Aa}}$ & $1,4^{A z}$ \\
\hline T3 & $S$ & 21,9 Аа & $1,4^{\mathrm{Aa}}$ & $8,2 \mathrm{Aa}$ & $6,3 \mathrm{Aa}$ & $2,2 \mathrm{Aa}$ & $1,4^{A} \mathrm{c}$ \\
\hline T4 & C & $24,3^{\mathrm{Aa}}$ & $1,6^{\mathrm{Aa}}$ & $9,1^{\mathrm{Aa}}$ & $5,5^{\mathrm{Aa}}$ & $2,4^{\mathrm{Aa}}$ & $1,3^{A c}$ \\
\hline T4 & $S$ & $25,6^{\mathrm{Aa}}$ & $1,4^{\mathrm{Aa}}$ & $9,1^{\mathrm{Aa}}$ & 7,4 $\mathrm{Aa}$ & $2,1^{\mathrm{Aa}}$ & $1,3^{A} \mathrm{c}$ \\
\hline \multicolumn{8}{|c|}{ Pastagem Nativa } \\
\hline T1 & C & $16,7^{\mathrm{Aa}}$ & $1,7^{\mathrm{Aa}}$ & $11,4^{\mathrm{Aa}}$ & $3,5 \mathrm{Aa}$ & $2,0^{\mathrm{Aa}}$ & $1,1^{A} \bar{c}$ \\
\hline T1 & $S$ & $15,1^{\mathrm{Aa}}$ & $1,5^{\mathrm{Aa}}$ & 11,9 Аa & 3,9 Aa & $2,6^{\mathrm{Aa}}$ & $1,3^{A c}$ \\
\hline $\mathrm{T} 2$ & C & $18,1^{\mathrm{Aa}}$ & $2,0^{\mathrm{Aa}}$ & $13,3^{\mathrm{Aa}}$ & $3,8^{\mathrm{Aa}}$ & $2,4^{\mathrm{Aa}}$ & $1,3^{A}$ \\
\hline $\mathrm{T} 2$ & $\mathrm{~S}$ & $15,1^{\mathrm{Aa}}$ & 1,3 Aa & $10,4^{\text {Aa }}$ & 3,9 Аa & 1,9 Aa & $1,2^{A c}$ \\
\hline T3 & C & $16,1^{\mathrm{Aa}}$ & $2,0^{\mathrm{Aa}}$ & $14,1^{\mathrm{Aa}}$ & $4,0^{\mathrm{Aa}}$ & $2,3^{\mathrm{Aa}}$ & $1,3^{A}$ \\
\hline T3 & $S$ & $17,2^{\mathrm{Aa}}$ & $1,5^{\mathrm{Aa}}$ & $11,4^{\mathrm{Aa}}$ & $4,5^{\mathrm{Aa}}$ & $2,2^{\mathrm{Aa}}$ & $1,2^{A c}$ \\
\hline T4 & C & $17,1^{\mathrm{Aa}}$ & $1,8^{\mathrm{Aa}}$ & $10,2^{\mathrm{Aa}}$ & $3,8 \mathrm{Aa}$ & $2,2^{\mathrm{Aa}}$ & $1,2^{A}$ \\
\hline T4 & $S$ & 15,9 Аа & $1,5 \mathrm{Aa}$ & $9,8 \mathrm{Aa}$ & $4,3 \mathrm{Aa}$ & $2,4 \mathrm{Aa}$ & $1,5 A^{A}$ \\
\hline
\end{tabular}

$\mathrm{AE}=$ Arranjo espacial; $\mathrm{A}=$ Adubação; $\mathrm{S}=\mathrm{Sem} ; \mathrm{C}=\mathrm{Com} ;{ }^{(1)}$ Letras maiúsculas referem-se ao teste de Tukey (arranjo espacial) e letras minúsculas (adubação) referem-se ao teste $t$ Student para separação dos contrastes de médias.

Quanto aos teores de micronutrientes (Tabela 4) é possível verificar valores superiores nos tratamentos com e sem adubação, tanto para o angico-vermelho (B e $Z n$ ), como para a pastagem nativa (Fe e $\mathrm{Zn}$ ). Porém os teores de micronutrientes também não diferem entre tratamentos. Os teores de micronutrientes se deram na ordem $\mathrm{Fe}>\mathrm{Mn}>\mathrm{Zn}>\mathrm{B}>\mathrm{Cu}$ para $P$. rigida e $\mathrm{Mn}>\mathrm{Fe}>\mathrm{Zn}>\mathrm{B}>\mathrm{Cu}$ para a pastagem nativa.

Em relação a nutrição vegetal para essências florestais, os teores de macronutrientes de $P$. rigida encontram-se dentro da faixa adequada, com exceção do $K$ que se encontra abaixo do nível crítico (MALAVOLTA et al., 1997). Os autores propõem as seguintes faixas de teores para macronutrientes $\left(\mathrm{g} \mathrm{kg}^{-1}\right)$ de: $\mathrm{N}$ de 12 a $35, \mathrm{P}$ de 1,0 a 2,3, K de 10 a 14, Ca de 3 a 12, Mg de 1,5 a 5,0.
Tabela 4. Teores médios de micronutrientes nas folhas de P.rigida e na biomassa da pastagem nativa nos diferentes tratamentos, aos 24 meses de idade.

\begin{tabular}{|c|c|c|c|c|c|c|}
\hline \multirow{2}{*}{$\mathrm{AE}$} & 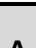 & B & $\mathrm{Cu}$ & $\mathrm{Fe}$ & $\mathrm{Mn}$ & $\mathrm{Zn}$ \\
\hline & A & \multicolumn{5}{|c|}{$\left(\mathrm{mg} \mathrm{kg}^{-1}\right)$} \\
\hline \multicolumn{7}{|c|}{ P. rigida } \\
\hline $\mathrm{T} 2$ & $C$ & $17,5^{\mathrm{Aba}^{1}}$ & $6,2^{\mathrm{Aa}}$ & $58,0^{\mathrm{Aa}}$ & $74,4^{\mathrm{Aa}}$ & $37,4^{\mathrm{Aa}}$ \\
\hline $\mathrm{T} 2$ & $S$ & $16,9^{\mathrm{Ba}}$ & $5,1^{\mathrm{Aa}}$ & $73,0^{\mathrm{Aa}}$ & 72,3 Аa & $21,0^{A b}$ \\
\hline T3 & C & $20,1^{\mathrm{ABa}}$ & $7,6^{\mathrm{Aa}}$ & 91,9 Аа & $74^{\mathrm{Aa}}$ & $38,4^{\mathrm{Aa}}$ \\
\hline T3 & $S$ & $19,6^{\mathrm{ABa}}$ & $5,6^{\mathrm{Aa}}$ & 68,8 Аa & 73,4 Аa & $38,4^{\mathrm{Aa}}$ \\
\hline T4 & C & $21,8^{\mathrm{ABa}}$ & $7,0^{\mathrm{Aa}}$ & 90,9 Аа & $70,2^{\mathrm{Aa}}$ & $25,1 \mathrm{Aa}$ \\
\hline T4 & $S$ & 23,1 Аa & 5,9 Aa & $76,2^{\mathrm{Aa}}$ & $68,1^{\mathrm{Aa}}$ & 20,9 Аа \\
\hline \multicolumn{7}{|c|}{ Pastagem nativa } \\
\hline $\mathrm{T} 1$ & $C$ & $18,1^{\mathrm{Aa}(1)}$ & $6,3^{\mathrm{Aa}}$ & $213,8^{\mathrm{Aa}}$ & $545,5 \mathrm{Aa}$ & $19,7^{\mathrm{Aa}}$ \\
\hline T1 & $\mathrm{S}$ & 18,0 Аа & $7,0^{\mathrm{Aa}}$ & $165,0^{\mathrm{Aa}}$ & $620,3 \mathrm{Aa}$ & $22,1 \mathrm{Aa}$ \\
\hline $\mathrm{T} 2$ & C & $18,0^{\mathrm{Aa}}$ & $7,2^{\mathrm{Aa}}$ & $222,0^{\mathrm{Aa}}$ & 733,4 Аа & $18,1^{\mathrm{Aa}}$ \\
\hline $\mathrm{T} 2$ & $S$ & $13,9 \mathrm{Aa}$ & $5,9 \mathrm{Aa}$ & $171,6 \mathrm{Aa}$ & $590,4 \mathrm{Aa}$ & $20,3^{A b}$ \\
\hline T3 & C & $15,1^{\mathrm{Aa}}$ & $7,5^{\mathrm{Aa}}$ & $125,4 \mathrm{Aa}$ & 518,4 Аa & $16,6^{\mathrm{Aa}}$ \\
\hline T3 & $S$ & $17,2^{\mathrm{Aa}}$ & $7,7^{\mathrm{Aa}}$ & $219,2^{\mathrm{Ab}}$ & $579,9 \mathrm{Aa}$ & $19,8^{\mathrm{Aa}}$ \\
\hline T4 & C & 13,9 Аa & $7,6^{\mathrm{Aa}}$ & $166,1^{\mathrm{Aa}}$ & $573,5 \mathrm{Aa}$ & $19,8^{\mathrm{Aa}}$ \\
\hline T4 & $S$ & $15,42 \mathrm{Aa}$ & $7,2^{\mathrm{Aa}}$ & 221,6 Аа & $506,3 \mathrm{Aa}$ & $18,2 \mathrm{Aa}$ \\
\hline
\end{tabular}

$\mathrm{AE}=$ Arranjo espacial; $\mathrm{A}=$ Adubação; $\mathrm{S}=\mathrm{Sem} ; \mathrm{C}=\mathrm{Com} ;{ }^{(1)}$ Letras maiúsculas referem-se ao teste de Tukey (arranjo espacial) e letras minúsculas (adubação) referem-se ao teste $t$ Student para separação dos contrastes de médias.

Resultados de $\mathrm{K}$ abaixo do nível crítico para a espécie florestal se deve, possivelmente a insuficiente fertilização potássica no sítio, uma vez que o solo apresentava teor baixo K (CQFS, 2016) na implantação. Considerando, a competição inicial com as árvores, a pastagem, pela sua maior taxa crescimento e maior abundância relativa de raízes finas, é mais eficiente no consumo de recursos do solo (DIAS-FILHO, 2006). Isso indica a necessidade de uma definição de fertilização considerando essa dinâmica, afim suprir adequadamente todos os componentes do consórcio.

Considerando a literatura disponível, indivíduos adultos de $P$. rigida em uma Floresta Estacional Decidual apresentaram teores de 23,3 $\mathrm{g} \mathrm{kg}^{-1}$ de $\mathrm{N}, 1,9 \mathrm{~g} \mathrm{~kg}^{-1}$ de $\mathrm{P}$, 4,8 $\mathrm{g} \mathrm{kg}^{-1}$ de $\mathrm{K}, 19,1 \mathrm{~g} \mathrm{~kg}^{-1}$ de Ca e 2,0 $\mathrm{g} \mathrm{kg}^{-1}$ de $\mathrm{Mg}$ (CUNHA, 1997). Já os teores de indivíduos de angicovermelho na arborização urbana, a espécie apresentou teores médios de macronutrientes na ordem $24,94 \mathrm{~g} \mathrm{~kg}$ ${ }^{1}$ de $\mathrm{N}, 0,97 \mathrm{~g} \mathrm{~kg}^{-1}$ de $\mathrm{P}, 6,28 \mathrm{~g} \mathrm{~kg}^{-1}$ de $\mathrm{K}, 11,58 \mathrm{~g} \mathrm{~kg}^{-1}$ de Ca, $1,62 \mathrm{~g} \mathrm{~kg}^{-1} \mathrm{de} \mathrm{Mg}$ e $1,15 \mathrm{~g} \mathrm{~kg}^{-1}$ de S (BRUN et al., 2012). De acordo com os autores, os teores médios de micronutrientes nas folhas de $P$. rigida foram: $33,04 \mathrm{mg}$ $\mathrm{kg}^{-1}$ de $\mathrm{B}, 8,66 \mathrm{mg} \mathrm{kg}^{-1}$ de $\mathrm{Cu}, 450,30 \mathrm{mg} \mathrm{kg}^{-1}$ de $\mathrm{Fe}$, $139,27 \mathrm{mg} \mathrm{kg}^{-1}$ de $\mathrm{Mn}$ e $19,17 \mathrm{mg} \mathrm{kg}^{-1}$ de $\mathrm{Zn}$ (BRUN et al., 2012).

Considerando ambos os trabalhos (CUNHA, 1997; 
BRUN et al., 2012), evidenciam-se os baixos teores de $\mathrm{Ca}$ obtidos no presente trabalho. Na implantação, o solo apresentava teores médios de Ca (CQFS, 2016), considerando que não houve calagem e os teores encontraram-se na faixa adequada (MALAVOLTA et al., 1997), a competição com a pastagem nativa não propiciou o maior acúmulo desse nutriente.

Geralmente o nitrogênio é o elemento com maior teor nas folhas das espécies vegetais (Tabela 3), entretanto, as espécies leguminosas fixadoras de $\mathrm{N}$ tendem serem superiores aos teores de outras espécies não leguminosas (FRANCO et al., 2003). Associada a essa informação, os teores de macronutrientes nas folhas de $P$. rigida demonstram o potencial da espécie no aporte de nutrientes, através da deposição de serapilheira (VOGEL et al., 2012). Esse aporte tanto de material orgânico, quanto de nutrientes pela espécie florestal, pode aumentar a produtividade da pastagem, do que é possível se obter ganhos econômicos futuros (VIERA, 2010).

Em relação à pastagem nativa, os teores de macronutrientes e micronutrientes se apresentaram dentro da faixa adequada em de comparação aos níveis críticos estabelecidos para gramíneas anuais (BISSANI et al., 2008). Como exemplo, para capim elefante (Pennisetum purpureum Schum) os autores citados recomendam faixa adequada em $\%(\mathrm{~m} / \mathrm{m})$ de, 1,5-25 de $\mathrm{N}, 0,1-0,3$ de $\mathrm{P}, 1,5-5,0$ de $\mathrm{K}, 0,3-0,8$ de $\mathrm{Ca}, 0,15-0,4$ de $\mathrm{Mg}$ e 0,1-0,3 de $\mathrm{S}$. Enquanto que para braquiária (Brachiaria (Trin.) Griseb) se recomenda em $\%(\mathrm{~m} / \mathrm{m}), 1,2-2,0$ de $N$, 0,1-0,3 de $\mathrm{P}, 1,2-2,5$ de $\mathrm{K}, 0,2-0,6$ de $\mathrm{Ca}, 0,15-0,4$ de $\mathrm{Mg}$ e 0,1-0,25 de $S$ (BISSANI et al., 2008).

Em relação aos micronutrientes, as faixas adequadas em $\mathrm{mg} \mathrm{kg}^{-1}$ indicados pelos autores para capim elefante são entre 10-25 de B, 4-17 de $\mathrm{Cu}, 50-200$ de $\mathrm{Fe}, 40-200$ de Mn e 20-50 de Zn (BISSANI et al., 2008). Para braquiária, os autores definem em mg kg-1, 10-25 de B, 4-12 de $\mathrm{Cu}$, 50-250 de Fe, 40-250 de Mn e 20-50 de Zn.

Não há diferenciação entre os teores obtidos na pastagem nativa (T1) em relação aos tratamentos com inserção do componente arbóreo, demonstrando que $P$. rigida não afeta negativamente o estado nutricional da pastagem. Inferência semelhante pode ser realizada em relação à densidade de indivíduos arbóreos, em função do arranjo espacial, que não interferiu nos aspectos nutricionais. Conforme a literatura, o sombreamento moderado, pela presença das árvores, não interfere na qualidade da pastagem, visto que proporciona aumento nos teores de N (PACIULLO et al., 2007) e nos valores proteicos da pastagem (PACIULLO et al., 2011).

Contudo, com base nos resultados da análise nutricional dos componentes arbóreo e campestre, até o momento não podem ser feitas maiores inferências sobre os tratos culturais aqui avaliado. Comumente os teores de nutrientes avaliados separadamente não conseguem discriminar tratamentos, sobretudo envolvendo a adubação (RANGEL-VASCONCELOS et al., 2016). De acordo com os autores, a quantificação de biomassa e estoque de nutrientes seriam avaliações mais adequadas na obtenção das respostas quanto aos tratamentos, sob a perspectiva da nutricional.

\section{CONCLUSÕES}

A adubação do sistema proporcionou maior crescimento em altura e DAC dos indivíduos de $P$. rigida.

No arranjo espacial de $2 \times 4(\mathrm{~T} 2)$ foi observado maior crescimento em altura e DAC dos indivíduos de $P$. rigida.

Os diferentes tratos culturais do sistema avaliados não influenciaram em diferenças no estado nutricional de $P$. rigida e da pastagem nativa.

\section{REFERÊNCIAS}

ALVARES, C.A. et al. Köppen's climate classification map for Brazil. Meteorologische Zeitschrift, v.22, n.6, p.711-728, 2013.

BISSANI, C.A. et al. Fertilidade dos solos e manejo da adubação de culturas. 2. ed. Porto Alegre: Metrópole, 2008.

BOLDRINI, I.I. A flora dos campos do Rio Grande do Sul. Campos Sulinos - conservação e uso sustentável da biodiversidade. Brasília: MMA, 2009.

BORGO, M. et al. Parapiptadenia rigida: angico. Espécies nativas da flora brasileira de valor econômico atual ou potencial: Planta para o futuro - região Sul. Brasília: MMA, 2011.

BRUN, E.J. et al. Avaliação nutricional de espécies nativas utilizadas na arborização do campus da Universidade Federal de Santa Maria-RS. Revista brasileira de arborização urbana, v.7, n.1, p.89-111, 2012.

BRUN, E.J. et al. Crescimento e acúmulo de serapilheira em plantios homogêneos de Luehea divaricata Mart. e Parapiptadenia rigida (Benth.) Brenan no Sudoeste do Paraná. Ecologia e Nutrição florestal, v.1, n.3, p.125-132, 2013.

CASTRO, A.C. et al. Sistema silvipastoril na Amazônia: ferramenta para elevar o desempenho produtivo de búfalos. Ciência Rural, v.38, n.8, p.2395-2402, 2008.

COMISSÃO DE QUÍMICA E FERTILIDADE DO SOLO - CQFS. Manual de adubação e calagem para os estados do Rio Grande do Sul e de Santa Catarina. 11 ed. Porto Alegre: Sociedade Brasileira de Ciência do Solo, 2016.

CUNHA, G.C. Aspectos da Ciclagem de nutrientes em diferentes 
fases sucessionais de uma Floresta Estacional do Rio Grande do Sul. 1997. 86p. (Dissertação de Mestrado).

DIAS-FILHO, M. B. Competição e sucessão vegetal em pastagens. 1 ed. Embrapa Amazônia Oriental: Pastagem, v.1, 2006. Disponível em: http://www.diasfilho.com.br/ Competicao_e_sucessao_em_pastagens.pdf

EMBRAPA. Sistema Brasileiro de Classificação de Solo. 5. ed. Brasília: EMBRAPA, 2018.

FRANCO, A.A. et al. Importância das leguminosas arbóreas na recuperação de áreas degradadas e na sustentabilidade de sistemas agroflorestais. 1 ed. Embrapa Pantanal: Sistemas agroflorestais, v.1, 2003. Disponível em: http://saf.cnpgc. embrapa.br/publicacoes/15.pdf

GASPARIN, E. et al. Supervivencia y crecimiento inicial de Parapiptadeniarigida em campo. Floresta, v.47, n.4, p.533-541, 2017.

GUADAGNIN, D.L. et al. Árvores e arbustos exóticos invasores no Pampa: questões ecológicas, culturais e sócio-econômicasde um desafio crescente. Campos Sulinos - conservação e uso sustentável da biodiversidade. Brasília: MMA, 2009.

JOSE, S.; DOLLINGER, J. Silvopasture: a sustainable livestock production system. Agroforestry systems, v.93, p.1-9, 2019.

LELES, P.S.S.; OLIVEIRA NETO, S.N.; ALONSO, J.M. Restauração florestal em diferentes espaçamentos. Recomposição Florestal da Bacia do Rio Guandu. Seropédica: Editora Rural, 2013.

LIMA, R. et al. Efeito do espaçamento no desenvolvimento volumétrico de Pinus taeda L. Floresta e Ambiente, v.20, n.2, p. 223-230, 2013.

LÓPEZ-SANTIAGO, J.G. et al. Carbon storage in silvopastoral system compared to that in a deciduous dry Forest in Michoacán, Mexico. Agroforestry systems, v.93, n.1, p.199-211, 2018.

LORENZI, H. Árvores Brasileiras: Manual de identificação e Cultivo de Plantas Arbóreas Nativas do Brasil. 5. Ed. Nova Odessa: Instituto Plantarum, 2008.

MALAVOLTA, E. et al. Avaliação do estado nutricional das plantas: princípios e aplicações. Piracicaba: Potafós, 1997.

MARCUZZO, S.B. et al. Plantio de espécies nativas para restauração de áreas em unidades de conservação: um estudo de caso no Sul do Brasil. Floresta, v.45, n.1, p.129-140, 2015.

MARTÍNEZ, J. et al. Silvopastoral systems enhance soil quality in grasslands of Colombia. Applied and Environmental Soil Science, v. 1, p.1-8, 2014.

NASCIMENTO, D.F. et al. Crescimento inicial de seis espécies florestais em diferentes espaçamentos. Cerne, v.18, n.1, p.159165, 2012.

OLIVEIRA NETO, S.N. et al. Crescimento e distribuição diamétrica de Eucalyptus camaldulensis em diferentes espaçamentos e níveis de adubação na região de Cerrado de Minas Gerias. Floresta, v.40, n.4, p.755-762, 2010.

PACIULLO, D.S.C. et al. Características produtivas e nutricionais do pasto em sistema agrossilvipastoril, conforme a distância das árvores. Pesquisa agropecuária brasileira, v.46, n.10, p.11761183, 2011.

PACIULLO, D.S.C. et al. Morfofisiologia e valor nutritivo do capim-braquiária sob sombreamento natural e a sol pleno. Pesquisa Agropecuária Brasileira, v.42, n.4, p.573-579, 2007.

PORFÍRIO-DA-SILVA, V. et al. Arborização de pastagens com espécies florestais madeireiras: implantação e manejo. 1 ed. Embrapa Florestas: Sistemas Agroflorestais, v.1, 2010. Disponível em: http://www.cnpf.embrapa.br/pesquisa/safs/ Cartilha_Arborizacao_final_2010.pdf

RANGEL-VASCONCELOS, L.G.T. et al. Acúmulo de biomassa e nutrientes de duas leguminosas arbóreas introduzidas em sistema de pousio na Amazônia. Ciência Florestal, v.26, n.3, p.735-746, 2016.

$R$ CORE TEAM. R: A language and environment for statistical computing. Vienna: R Foundation for Statistical Computing, 2016.

RIBASKI, J. et al. Sistemas silvipastoris: Estratégias para o desenvolvimento rural sustentável para a metade Sul do estado do Rio Grande do Sul. Ed 1. Embrapa Floresta: Sistemas Agroflorestais, v.1, 2005. Disponível em: https://core.ac.uk/ download/pdf/15437199.pdf

SCHEER, M.B. et al. Substratos à base de lodo de esgoto compostado na produção de mudas de Parapiptadenia rigida (Benth.) Brenan. Scientia Forestalis, v.38, n.88, p.637-644, 2010.

SILVA, T.C. et al. Determinação dos teores de taninos na casca de galhos de três espécies arbóreas da caatinga. $3^{\circ}$ Congresso Brasileiro de Ciência e Tecnologia da Madeira, p.1-6, 2017.

TEDESCO, M.J. et al. Análises de solo, plantas e outros materiais.2 ed. Porto Alegre: UFRGS, 1995.

VIERA, M.; SCHUMACHER, M.V. Biomassa em povoamentos monoespecíficos e mistos de eucalipto e acácia-negra e do milho em sistema agrossilvicultural. Revista Cerne, v.17, n.2, p.259265, 2011.

VIERA, M. Crescimento inicial e produtividade em plantios monoespecíficos e mistos de Eucalyptus urograndis e Acaciamearnsii em sistema agrossilvicultural. 2010. 140p. (Dissertação de Mestrado).

VOGEL, H.L.M. et al. Deposição de serapilheira e nutrientes por espécies nativas em uma Floresta Estacional Decidual em Itaara, RS, Brasil. Floresta, v.42, n.1, p.129-136, 2012.

Recebido em 07-10-2019 Aceito em 13-01-2020 